\title{
Essential laminations and Haken normal form: laminations with no holonomy
}

\author{
MARK BRITTENHAM
}

In [Br1] we showed how, given a triangulation $\tau$ of a 3-manifold $\mathrm{M}$, to use an essential lamination $\mathcal{L}$ in $\mathrm{M}$ to find a (usually different) essential lamination $\mathcal{L}_{0}$ which is in Haken normal form with respect to the triangulation $\tau$. This involved an (in principle) infinite sequence of isotopies, to 'grow' $\mathcal{L}_{0}$ out of stable portions of $\mathcal{L}$. In this paper we show that if the lamination $\mathcal{L}$ has no holonomy (see $[\mathrm{Re}]$ ), then $\mathcal{L}_{0}=\mathcal{L}$; that is, this infinite isotopy is in fact a finite one - the isotopy process halts after finite time.

Theorem 1. If $\tau$ is a triangulation of the 3-manifold $M$, and $\mathcal{L} \subseteq M$ is an essential lamination in $M$ with no holonomy, then $\mathcal{L}$ may be isotoped to be in Haken normal form w.r.t. $\tau$.

Hatcher [Ha] has shown that an incompressible measured lamination can be put into Haken normal form w.r.t. a triangulation. Since such laminations have no holonomy, the above result gives a different proof that these laminations can be put into normal form.

\section{Idea of the proof.}

We will say that an essential lamination $\mathcal{L} \subseteq M$ is in Haken normal form w.r.t. a triangulation $\tau$ of $\mathrm{M}$ if $\mathcal{L}$ intersects $\tau$ transversely, and, for every 3 -simplex $\Delta^{3}$ of $\tau, \mathcal{L} \cap \Delta^{3}$ consists of normal disks. In [Br1], we defined a sequence of isotopies which serve as the most 'natural' way to attempt to push a given essential lamination $\mathcal{L}$, transverse to a triangulation, into Haken normal form. For completeness, we give a description of this procedure here.

The idea is to work a single 3 -simplex $\Delta^{3}$ at a time, and then create the sequence of isotopies by running cyclically through the 3 -simplices $\Delta_{1}^{3}, \ldots, \Delta_{\mathrm{n}}^{3}$ of $\tau$. Each isotopy in the sequence is itself a finite sequence of 'simple' isotopies, whose sole purpose is to push all of the 'problems' with $\mathcal{L} \cap \Delta^{3}$ out of $\Delta^{3}$, and, in so doing, making $\mathcal{L}$ meet $\Delta^{3}$ in a collection of normal 



Figure 1a.

disks. More precisely, each simple isotopy is either a compression or a $\partial$ compression.

A compression consists of compressing $\mathcal{L}$ along a family of loops of $\mathcal{L} \cap \partial \Delta^{3}$, which, after throwing out the 2-spheres that this must inevitably create, can be thought of as an isotopy of $\mathcal{L}$. For simplicity we adopt the strategy of thinking of compression as a surgery along all of the loops of $\mathcal{L} \cap \partial \Delta^{3}$, as in Figure 1a. It should be clear, though, that this process, applied to a normal disk (and, in fact, to any disk leaf of $\mathcal{L} \cap \Delta^{3}$ ), gives a sphere in the 3-simplex, which we throw away, and the same normal disk back again. So normal disks only 'wiggle' during a compression; a more careful approach would allow us to insist that normal disks remain fixed, while all those loops needing compressing (i.e., those that do not bound disk leaves of $\mathcal{L} \cap \Delta^{3}$ ) are compressed. A $\partial$-compression is an isotopy which pushes $\mathcal{L}$ along a disk in $\Delta^{3}$, whose boundary consists of an arc in a leaf of $\mathcal{L}$ and an $\operatorname{arc}$ in $\tau^{(1)} ;$ see Figure $1 \mathrm{~b}$.

A finite application of these two isotopies will make $\mathcal{L}$ meet $\Delta^{3}$ in a collection of normal disks. We denote the result of the simple isotopies making $\mathrm{I}_{\mathrm{r}-1}(\mathcal{L})$ meet the $\mathrm{r}$-th 3 -simplex in normal disks (where, since we continue through the 3 -simplices cyclically, we really mean $r(\bmod n)) I_{r-1, r}$, and set $I_{r-1, r} \circ I_{r-1}=I_{r}$. So $I_{r}(\mathcal{L})$ is the result of isotoping $\mathcal{L}$ to meet the first $\mathrm{r} 3$-simplices, in turn, in normal disks.

What makes this sequence of isotopies useful, and the main property that both the proof in $[\mathrm{Br} 1]$ and the proof we give here exploit, is that these isotopies are not altering $\mathcal{L} \cap \tau^{(1)}$ in any significant way - for each of the isotopies $\mathrm{I}, \mathrm{P}=\mathrm{I}(\mathcal{L}) \cap \tau^{(1)} \subseteq \mathcal{L} \cap \tau^{(1)}$, and I is constant on P. In other words, 

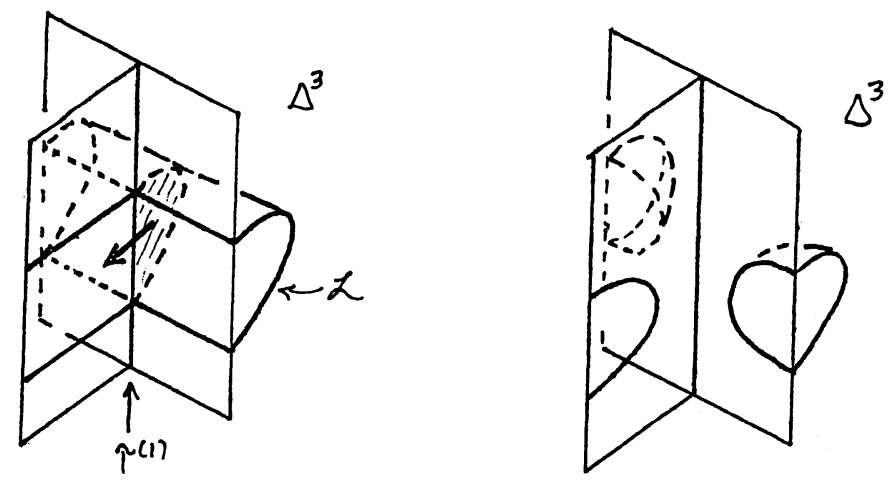

Figure 1b.

the isotopy I can only remove points from $\mathcal{L} \cap \tau^{(1)}$, not create new ones, and it doesn't move any of the points it doesn't remove. This is easily seen to be the case by considering the effect of each of the simple isotopies on $\mathcal{L} \cap \tau^{(1)}$.

This leads us to consider the set of points $C \subseteq \mathcal{L} \cap \tau^{(1)}$, the set of stable points, which never move under any of the isotopies. $\mathrm{C}$ is the intersection of the nested sequence of closed sets $\mathrm{I}_{\mathrm{r}}(\mathcal{L}) \cap \tau^{(1)}$. The proof of [Br1] was to show that around each of these stable points, stable normal disks would grow; at some point, the normal disk containing a stable point would itself stabilize, and remain fixed under all further isotopies. In other words, a new lamination would grow out of these stable points, being created from the 'eventually stable' parts of the original lamination $\mathcal{L}$. However, these eventually stable parts would not themselves form a lamination. The set $\mathrm{X}$ of stable disks meets $\tau^{(1)}$ in the closed set C, but X itself need not be a closed set, the problem being that some normal disks are 'missing', where different normal disk types in a 3-simplex come together; see Figure 2. Much of [Br1] involved very carefully turning $\mathrm{X}$ into a lamination. The main purpose of this paper is to show that in the absence of holonomy, this last part will not be needed. This will follow readily from the following:

Proposition 2. If $\mathcal{L}$ has no holonomy, then the set of points $B=$ $\left(\mathcal{L} \cap \tau^{(1)}\right) \backslash C$ is a closed subset of $\left(\mathcal{L} \cap \tau^{(1)}\right.$, hence of $) \tau^{(1)}$.

The proof of this proposition constitutes the bulk of this paper.

Before proving the proposition in sections 2 and 3 , we will show how this result implies the main result of the paper. The points of $B=\left(\mathcal{L} \cap \tau^{(1)}\right) \backslash C$ are, by definition, the unstable points of the isotopies, so each is erased from $\mathcal{L} \cap \tau^{(1)}$ at some finite stage $\mathrm{I}_{\mathrm{r}}$ of the isotopies. But since $\mathrm{B}$ is a closed 


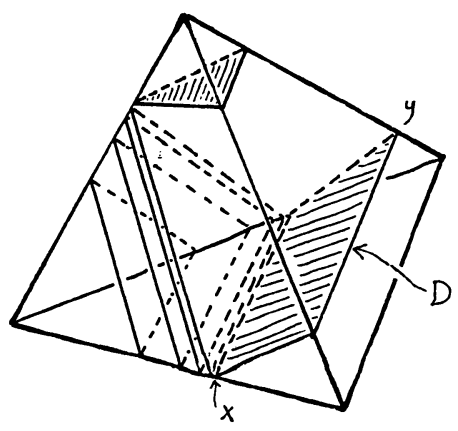

Figure 2.

set, so is every $\mathrm{B}_{\mathrm{r}}=\left(\mathrm{I}_{\mathrm{r}}(\mathcal{L}) \cap \tau^{(1)}\right) \backslash \mathrm{C}=\mathrm{I}_{\mathrm{r}}(\mathcal{L}) \cap\left(\left(\mathcal{L} \cap \tau^{(1)}\right) \backslash \mathrm{C}\right)=\mathrm{I}_{\mathrm{r}}(\mathcal{L}) \cap \mathrm{B} \subseteq \tau^{(1)}$. Also, the $B_{\mathrm{r}}$ are nested, since the $\mathrm{I}_{\mathrm{r}}(\mathcal{L}) \cap \tau^{(1)}$ are, and $\cap\left(\left(\mathrm{I}_{\mathrm{r}}(\mathcal{L}) \cap \tau^{(1)}\right) \backslash \mathrm{C}\right)$ $=\left(\cap\left(\mathrm{I}_{\mathrm{r}}(\mathcal{L}) \cap \tau^{(1)}\right)\right) \backslash \mathrm{C}=\mathrm{C} \backslash \mathrm{C}=\emptyset$, so since $\tau^{(1)}$ is compact, we must have $\left(\mathrm{I}_{\mathrm{r}}(\mathcal{L} \cap) \tau^{(1)}\right) \backslash \mathrm{C}=\emptyset$ for some $\mathrm{r}$, i.e., $\mathrm{I}_{\mathrm{r}}(\mathcal{L}) \cap \tau^{(1)} \subseteq \mathrm{C}$, so,since $\mathrm{C} \subseteq \mathrm{I}_{\mathrm{r}}(\mathcal{L}) \cap \tau^{(1)}$, $\mathrm{I}_{\mathrm{r}}(\mathcal{L})=\mathrm{C}$. So all of the unstable points disappear at some finite time $\mathrm{r}$. But then:

Lemma 3. $\mathrm{I}_{\mathrm{r}+\mathrm{n}}(\mathcal{L})$ is in normal form w.r.t. $\tau$.

Proof. We need to show that for each 3-simplex $\Delta_{\mathrm{i}}^{3}, \mathrm{i}=1, \ldots, \mathrm{n}$, of $\tau, \mathrm{I}_{\mathrm{r}+\mathrm{n}}(\mathcal{L})$ meets $\Delta_{\mathrm{i}}^{3}$ in disks, and that each disk of $\mathrm{I}_{\mathrm{r}+\mathrm{n}}(\mathcal{L}) \cap \Delta_{\mathrm{i}}^{3}$ meets each 1-simplex of $\Delta_{\mathrm{i}}^{3}$ at most once. For the first, if some circle of $\mathrm{I}_{\mathrm{r}}(\mathcal{L}) \cap \partial \Delta_{\mathrm{i}}^{3}$ doesn't bound a disk of $I_{\mathrm{r}}(\mathcal{L}) \cap \Delta_{\mathrm{i}}^{3}$, then compressing along (a smaller sphere parallel to) $\partial \Delta_{\mathrm{i}}^{3}$ must create spheres in $\mathrm{I}_{\mathrm{r}}(\mathcal{L})$ that get thrown away, as in Figure 1a. These newly created spheres cannot meet $\tau^{(1)}$; if they did, removing them would remove points of $\mathrm{I}_{\mathrm{r}}(\mathcal{L}) \cap \tau^{(1)}=\mathrm{C}$, contradicting the fact that these are all stable points of the isotopies. After doing this for each 3 -simplex, we will succeed in making $\mathrm{I}_{\mathrm{r}+\mathrm{n}}(\mathcal{L})$ meet each 3-simplex in disks, provided we have not done any $\partial$-compressions in the meantime. But a $\partial$-compression would remove points of $\mathrm{I}_{\mathrm{r}}(\mathcal{L}) \cap \tau^{(1)}=\mathrm{C}$, which is impossible, since these points are stable. So $I_{r+n}(\mathcal{L}) \cap \Delta_{i}^{3}$ consists of disks. Each of these disks must then meet each 1-simplex of $\partial \Delta_{\mathrm{i}}^{3}$ at most once, since if they didn't we would find a $\partial$-compression to carry out, which, again, would be forced to remove stable points, an impossibility. So $\mathrm{I}_{\mathrm{r}+\mathrm{n}}(\mathcal{L})$ is in Haken normal form w.r.t. $\tau$. 


\section{The limiting surface.}

We wish to show that if $\mathcal{L}$ is an essential lamination with no holonomy and $I_{k}$ is the sequence of isotopies attempting to put $\mathcal{L}$ into Haken normal form w.r.t the triangulation $\tau$, then $\mathrm{B}=\left(\mathcal{L} \cap \tau^{(1)}\right) \backslash \mathrm{C}$ is a closed set, i.e., (since $\mathcal{L} \cap \tau^{(1)}=\mathrm{B} \cup \mathrm{C}$ is closed) no point of $\mathrm{C}$ is a limit point of $\mathrm{B}$. We will argue by contradiction. Let $A \subseteq C$ be the set of stable points (i.e., points of C) that are limited on by unstable points (i.e., points of B). In other words, $A$ is the set of limit points of $B$ which are contained in $C$ (i.e., $A=\bar{B} \cap C$ ). We will show that in general this is a finite set, hence is contained in a finite number of stable disks of some $I_{k}(\mathcal{L})$, the union of which is then a compact surface $\mathrm{S}$. Then we will show that it is impossible to have both $A \neq \emptyset$ and have no holonomy around every loop in S.

Proposition 4. $A$ is finite.

Proof. The idea behind the proof is that our 'problems' cannot get too close to one another. The main tool in showing this is:

Lemma 5. If $\mathcal{L} \subseteq N(W)$ is an essential lamination carried by the essential branched surface $W$, and $\alpha$ is an arc of an I-fiber of $N(W)$ whose endpoints are contained in a leaf $L$ of $\mathcal{L}$, then $\alpha$ cannot be homotoped rel endpoints into $L$.

Proof. This follows immediately from [G-O, Theorem 1(d)], since $\alpha$ is an efficient arc for W.

We make use of this lemma by choosing an essential branched surface W carrying $\mathcal{L}$ and transverse to $\tau^{(1)}$, so that $\mathrm{N}(\mathrm{W}) \cap \tau^{(1)}$ consists of a finite number of arcs, each contained in an I-fiber of $\mathrm{N}(\mathrm{W})$. Then we choose a number $\epsilon$ so that any two points of $\mathcal{L} \cap \tau^{(1)}$ within $\epsilon$ of one another are contained in the same I-fiber of $\mathrm{N}(\mathrm{W}) \cap \tau^{(1)}$. This then means that no subarc of $\tau^{(1)}$ joining points of $\mathcal{L} \cap \tau^{(1)}$ and of length less than $\epsilon$ can be homotoped rel endpoints into a leaf of $\mathcal{L}$, by the lemma.

Now suppose that $A \subseteq \tau^{(1)}$ is infinite; it then meets some 1-simplex $\Delta_{i}^{1}$ in an infinite set, so $A$ has a limit point $x \in \Delta_{i}^{1}$. Because $A \subseteq C$ and $C$ is closed, $x \in \mathrm{C}$. So there exist points $a, b, c \in \mathrm{A}$, all in $\Delta_{\mathrm{i}}^{1}$, which are within $\epsilon / 2$ of $x$, hence are within $\epsilon$ of one another; see Figure 3. We may assume $b$ is between $a$ and $c$ on $\Delta_{\mathrm{i}}^{1}$. Then since $b$ is a limit point of $\mathrm{B}$, there are 


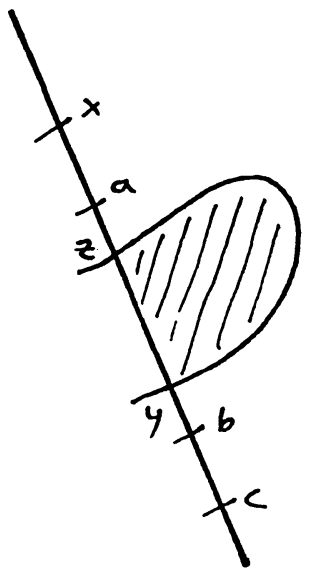

Figure 3.

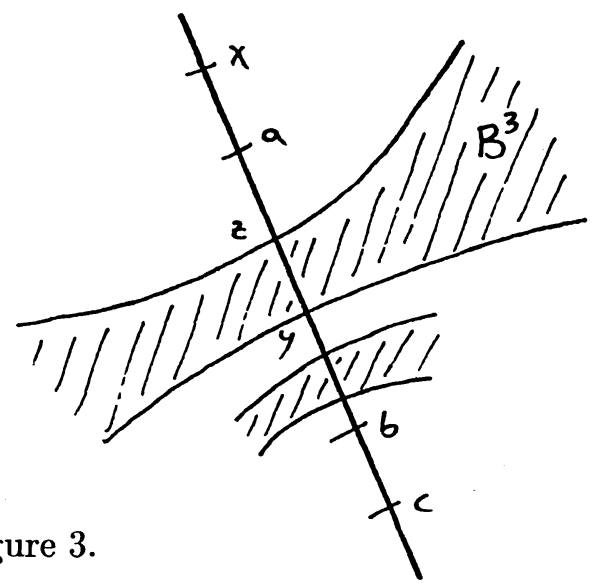

points of B lying arbitrarily close to $b$, on one side or the other, say the $a$-side. So in particular there is a point $y$ of B between $a$ and $b$. Since it is unstable, this point $y$ must be removed at some stage of the isotopies, say in the isotopy $\mathrm{I}_{\mathrm{k}, \mathrm{k}+1}$. This point then is either removed by a $\partial$-compression, or by removing a sphere surgered off of $\mathrm{I}_{\mathrm{k}}(\mathcal{L})$; see Figure 3 .

But in the first case we then get an arc in a leaf of $\mathrm{I}_{\mathrm{k}}(\mathcal{L})$, and in some 3 -simplex of $\tau$, one of whose endpoints is $y$, realizing the $\partial$-compression. This arc must have its other endpoint $z$ also in the interval between $a$ and $b$, since otherwise the interval between $y$ and $z$ will contain either $a$ or $b$. But then the $\partial$-compression will erase everything in $\mathrm{I}_{\mathrm{k}}(\mathcal{L}) \cap \tau^{(1)}$ between $y$ and $z$, which is impossible, since this will include either $a$ or $b$, which are both stable (hence cannot be erased). The interval between $y$ and $z$ consequently has length less than $\epsilon$, so the $\partial$-compressing arc in $\mathrm{I}_{\mathrm{k}}(\mathcal{L})$ between $y$ and $z$ gives an arc which, flowing back by the isotopies used to create $\mathrm{I}_{\mathrm{k}}$, gives an $\operatorname{arc}$ in $\mathcal{L}$ which is homotopic rel endpoints to a subinterval of $\Delta_{\mathrm{i}}^{1}$ of length less than $\epsilon$, contradicting Lemma 5.

In the second case the point $y$ is eventually (after a surgery) contained in a sphere $S^{2} \subseteq I_{k, k+1}(\mathcal{L})$ which we are going to erase. But since $M$ is irreducible [G-O], this sphere bounds a ball $\mathrm{B}^{3}$; in particular, this sphere separates M. Both $a$ and $b$ must be in the same component of $\mathrm{M} \mid \mathrm{S}^{2}$, since otherwise one of them is in $\mathrm{B}^{3}$, and hence is contained in a 2-sphere, since after a surgery the lamination consists of something isotopic to $\mathcal{L}$ together with a collection of spheres, and no leaf of $\mathcal{L}$ can be contained in the interior of a ball. But this sphere will be erased, contradicting the fact that $a$ and $b$ are both stable. So the sphere leaf containing $y$ does not separate $a$ and $b$, so it must meet the interval of $\Delta_{\mathrm{i}}^{1}$ between $a$ and $b$ an even number of 
times; in particular, it meets this interval in a point $z \neq y$ s.t. the interval between $y$ and $z$ is entirely contained in $\mathrm{B}^{3}$. But then we can choose any arc in $\mathrm{S}^{2}$ joining $y$ and $z$ and missing the (images of the) compressing disks used to cut $\mathrm{S}^{2}$ off of $\mathrm{I}_{\mathrm{k}}(\mathcal{L})$; this is possible since the compressing disks are just 'fat points' in $\mathrm{S}^{2}$. We then get an arc (which can be thought of as lying in $\left.\mathrm{I}_{\mathrm{k}}(\mathcal{L})\right)$ which is homotopic rel endpoints to the interval of $\Delta_{\mathrm{i}}^{1}$ between $y$ and $z$, since both arcs are contained in the ball $\mathrm{B}^{3}$. Since the interval between $y$ and $z$ is contained in the interval between $a$ and $b$, it has length less than $\epsilon$. But this once again contradicts Lemma 5 .

Therefore, in either case we arrive at a contradiction; therefore A is finite.

Note that the proof aboves tells us also that any two stable points that are less than $\epsilon$ apart can never have any unstable points between them such points could never be made to go away. This implies in particular that the points of $\mathrm{A}$ are isolated in $\mathrm{C}$ on the sides in which $\mathrm{B}$ limits on them.

Now since A is a finite set of stable points, it meets only finitely-many stable disks; we can, for convenience, split $\mathcal{L}$ open along the (finitely-many) leaves whose image under the isotopies contains these disks. This is to insure that these disks are limited upon on only one side by unstable disks; we could instead, in what follows, 'double-count' any of the points of A that are limited upon on both sides by points of $\mathrm{B}$, as well as the stable disks that contain them. The union of the disks containing this doubled version of $\mathrm{A}$ is a (not necessarily connected) compact surface $\mathrm{S}$, possibly with points on it's boundary identified; see Figure 4 . This surface $S$ is contained in $I_{k}(\mathcal{L})$ for some $\mathrm{k}$, since finitely-many stable disks will stabilize in finite time. Some points $x$ of $\mathrm{S} \cap \tau^{(1)}$ are not in A; but since such a point is in a stable normal disk $\mathrm{D}$ that does contain a point $y$ in $\mathrm{A}$, it must be a limit point of $\mathcal{L} \cap \tau^{(1)}$ from the side that $y$ is limited on. This is because, when the lamination is normal in the 3 -simplex containing $\mathrm{D}$, we must see a sequence of normal disks limiting on $\mathrm{D}$ from the side that $y$ is limited upon, since we see this at one of its corners. Therefore $\mathrm{x}$ must be a limit point of $\mathrm{C}$. But since on one end of $\mathrm{D} y$ is a limit point of $\mathrm{B}$ while on the other end $x$ is a limit point of $\mathrm{C}$, we can conclude that $\mathrm{x}$ lies at the interface of different stable normal disk types, as in Figure 2. This is because the points of $\mathrm{C}$ limiting on $x$ are eventually contained in stable disks. These stable disks cannot be limiting down on $\mathrm{D}$, since then $y$ would be limited upon by points of $\mathrm{C}$, contradicting the fact that it is limited upon by B. So these stable disks are not normally isotopic to D (i.e., they are not of the same normal disk type as D). 


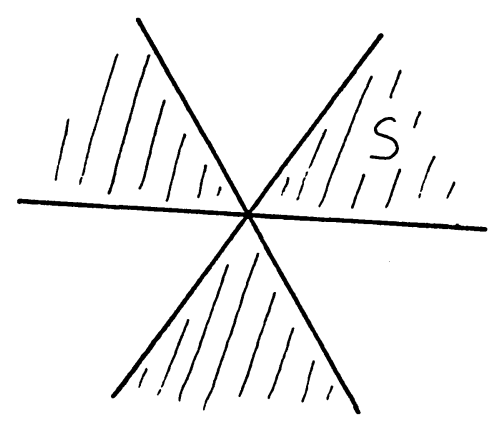

Figure 4 .

Points of $\mathrm{A}$ are interior points of $\mathrm{S}$, by the definition of $\mathrm{S} ; \mathrm{S}$ contains all of the (stable) normal disks that contain these points. So the only way that the compact surface $\mathrm{S}$ can fail to be closed is if it has boundary lying at the interfaces of different stable normal disk types (in the language of [Br1], if we were to take the closure $\overline{\mathrm{X}}$ of the union $\mathrm{X}$ of the stable disks, its singular set would contain $\partial \mathrm{S}$ ). What we will now show is that this picture, together with the fact that the leaves of $\mathcal{L}$ near $\mathrm{S}$ contain unstable points, and so keep being pushed and are stripped away, is inconsistent with the hypothesis of no holonomy (in $\mathcal{L}$, hence) around loops in $\mathrm{S}$.

\section{The mechanics of infinite pushability.}

Now we wish to introduce into this setting the fact that $\mathcal{L}$ has no holonomy, so there is, in particular, no holonomy in $\mathrm{I}_{\mathrm{k}}(\mathcal{L})$ along $\mathrm{S}$. Therefore, leaves of $I_{k}(\mathcal{L})$ passing sufficiently close to $S$ meet the normal fence over $S$ in surfaces, made up of normal disks, which are homeomorphic to $\mathrm{S}$ (see $[\mathrm{Re}])$, and, in fact, the normal projection of each of these surfaces onto $\mathrm{S}$ is a homeomorphism. What we must now ask ourselves is: How can any of these surfaces near $\mathrm{S}$ move? We know they must, by hypothesis (they meet $\tau^{(1)}$ in points of $B$, because $\left.A \neq \emptyset\right)$, but, it turns out, they can't. There is no way to start.

$\partial S \neq \emptyset$, since otherwise $S$ is a leaf of $\mathrm{I}_{\mathrm{r}}(\mathcal{L})$, so the nearby leaves lying above $\mathrm{S}$ are (homeomorphic to $\mathrm{S}$, so) are compact and made up of normal disks. But they are then stable, since the isotopies do not move normal disks. This contradicts the fact that they meet $\tau^{(1)}$ in points of B. 


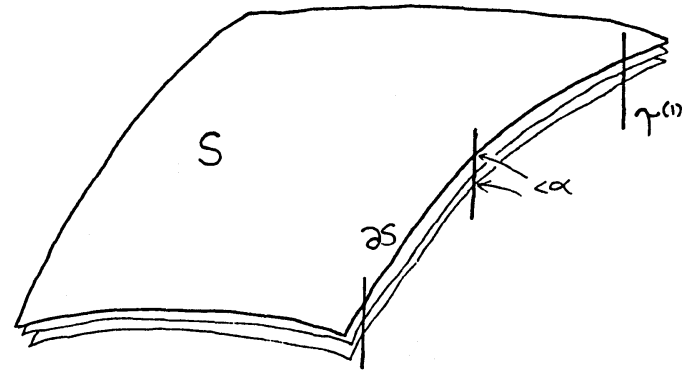

Figure 5.

$\partial \mathrm{S}$ meets $\tau^{(1)}$ in a finite number of points, all limited upon by points of C. For each, we can choose a $\delta<\epsilon$ so that there is a point of $\mathrm{C}$ within $\delta$ of the point on the limited sided of S. Then choose a number $\alpha$ less than all of these $\delta$ 's so that leaves passing within $\alpha$ of S meet the normal fence over $\mathrm{S}$ in surfaces homeomorphic to $\mathrm{S}$. Then the boundaries of these surfaces are all stable; they meet $\tau^{(1)}$ in points of $\mathrm{C}$; see Figure 5 . This is because the points of $\partial \mathrm{S} \cap \tau^{(1)}$ are all in $\mathrm{C}$, and are limited upon by points of $\mathrm{C}$, and once we are within $\alpha$ of $\partial \mathrm{S} \cap \tau^{(1)}$, we are between two points of $\mathrm{C}$ which are less than $\epsilon$ apart, so we must be in $\mathrm{C}$ by the argument above.

So the boundaries of these nearby surfaces are all stable; they never move. So how can any of the surfaces move? They consist of normal disks, since they are parallel to $\mathrm{S}$ and lie close to $\mathrm{S}$, which consists of normal disks. They cannot be initially pushed by a $\partial$-compression - this requires the points being erased to be contained in something which both isn't normal, which means we're at the boundary of the surface, and unstable, which the boundary isn't. So the only way that these surfaces can move is by compression. That is, we must be surgering along a sphere parallel to some $\partial \Delta_{\mathrm{i}}^{3}$ and removing the spheres in $\mathrm{I}_{\mathrm{k}}(\mathcal{L})$ this creates. These removed spheres must then include pieces of our surfaces near $\mathrm{S}$ - otherwise nothing near $\mathrm{S}$ would move. But the loops that we are compressing along cannot meet any of these surfaces, because these surfaces are made up of normal disks; when you compress a normal disk, the normal disks remain, so nothing would be removed from near $\mathrm{S}$. So it must be the case that a surface lying above $\mathrm{S}$ ends up completely contained in spheres that are erased. But then their boundaries also end up in these spheres, so are erased. This is impossible, since the boundaries contain stable points. 

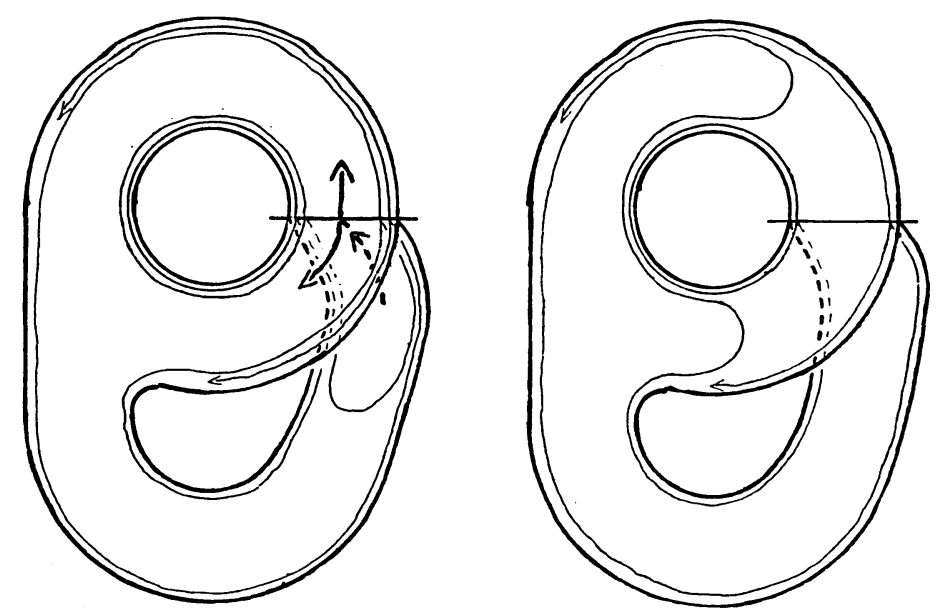

Figure 6.

So there is no way to move these surfaes lying above $\mathrm{S}$, so they are stable, but this contradicts the fact that they meet $\tau^{(1)}$ in unstable points. This gives us the required contradiction, so $A=\emptyset ;\left(\mathcal{L} \cap \tau^{(1)}\right) \backslash \mathrm{C}$ is closed.

\section{Closing observations.}

What we have shown is that in the absence of holonomy, there is no mechanism for removing those unstable portions of the lamination $\mathcal{L}$ which might limit on stable disks. We include a figure (see Figure 6) which shows that this need not be the case for a lamination with non-trivial holonomy; one can, with holonomy, continually recreate $\partial$-compressing disks, for example, which will provide a way to keep removing unstable points from the vicinity of stable points. The figure shows how a portion of the lamination might intersect the 2-skeleton of $\tau$; we do not know if this particular figure can be realized by an essential lamination in a 3-manifold; although the '1-dimensional lamination' in the 2-complex pictured has $\pi_{1}$-injective, end injective leaves.

It is worth noting that the techniques of this paper apply equally well to the process [Br2] of finding an essential lamination in normal form w.r.t. a regular cell decomposition of $M$. If the lamination one starts with has no holonomy, then at each stage of the infinite sequence of isotopies in [Br2] the 

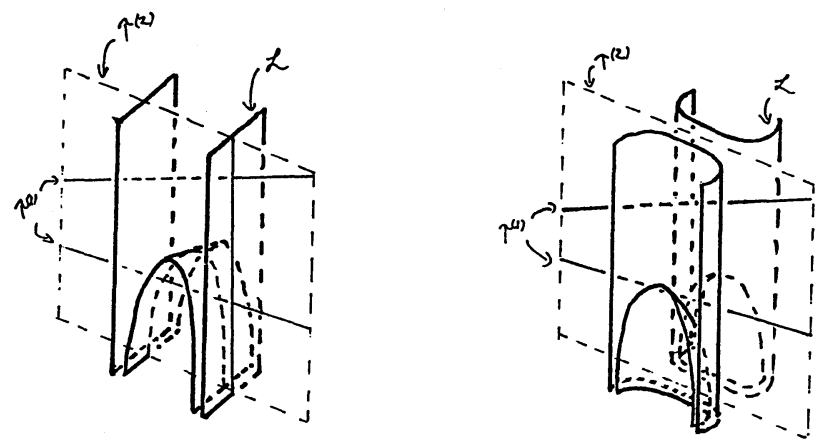

Figure 7.

lamination we get is isotopic to the one we started with, and each isotopy finishes in finite time. Therefore, the infinite collection of isotopies is really just a single infinite isotopy attempting to put the lamination into normal form w.r.t. the cell decomposition. But then the arguments above can be applied to the set of stable points (of this single sequence of isotopies) which are limited upon by unstable points, to show that such points do not exist therefore, eventually the isotopies stop doing anything, and our lamination is in normal form w.r.t. the cell decomposition. So we have:

Theorem 6. If $M$ is a 3-manifold with a regular cell decomposition $\left\{\mathrm{B}_{\mathrm{k}}^{3}\right\}$, and $\mathcal{L}$ is an essential lamination in $M$ with no holonomy, then $\mathcal{L}$ is isotopic to a lamination which is in Haken normal form w.r.t. the cell decomposition.

The arguments given here also provide us with a picture of how, in general, the sequence of isotopies, which finds a lamination in Haken normal form w.r.t a triangulation, behaves. Eventually, all unstable points become concentrated around the compact stable surface $\mathrm{S}$; all other unstable points, by the argument of section 1, disappear in finite time, since $\mathrm{C}$ together with a small open (in $\mathcal{L} \cap \tau^{(1)}$ ) neighborhood of $\mathrm{A}$ gives a relatively open set in $\mathcal{L} \cap \tau^{(1)}$ whose complement is then relatively closed and consists of unstable points, which therefore all disappear in finite time. So all of the 'action' of the isotopy becomes concentrated in a compact piece of $\mathrm{M} \mid \overline{\mathrm{X}}$ (in the terminology of $[\mathrm{Br} 1])$.

With this better picture of the isotopy process it might be possible to 'find' the limiting normal lamination in finite time, and, usually, this should perhaps be a sublamination of $\mathcal{L}$. In Figure 6, for example, the 'boundary' of the figure is a sublamination that is stable. One problem is that this 



Figure 8.

isotopy process will perform 'Reeb surgery' on a Reeb annulus lying in the 2-skeleton of $\mathrm{M}$ which also misses the 0 -skeleton (so long as it remains, i.e., the boundary of the annulus is stable); see Figure 7. So, for example, we can create 'accidental' Reeb components for the limiting normal lamination, by continually pushing the end of a 'half-Reeb' component (i.e., the neighborhood of a loop, in a leaf, having non-trivial holonomy) infinitely often; see Figure 8. The resulting normal lamination, with the Reeb component thrown away, is isotopic to the original one. The situation pictured here is easy to arrange, for any lamination with non-trivial holonomy, by choosing the right (or wrong?) triangulation of the ambient manifold. So the (admittedly simple) isotopy process we employ here cannot always 'find' a normal sublamination on its own, in finite time.

\section{References.}

[Br1] Brittenham, Essential laminations and Haken normal form, Pac. J. Math. 168 (1995), 217-234.

[Br2] Brittenham, Essential laminations and Haken normal form: regular cell decompositions, preprint.

[G-O] Gabai and Oertel, Essential laminations in 3-manifolds, Annals of Math., 130 (1989) 41-73.

[Ha] Hatcher, Measured laminations in 3-manifolds, preprint. 
[Re] Reeb, Sur certaines propriétés topologiques des variétés feuilletées, Actualité Sci. Indust. 1183, Hermann, Paris (1952).

RECEIVEd May 24Th, 1994.

DEPARTMENT OF MATHEMATICS

RLM 8.100

University of TeXas at Austin

Austin, TX 78712 